\title{
Lidil
}

Revue de linguistique et de didactique des langues

$64 \mid 2021$

Le passif dans la langue parlée

\section{Présentation. - Le passif dans la langue parlée}

\section{Badreddine Hamma}

\section{OpenEdition}

Journals

Édition électronique

URL : https://journals.openedition.org/lidil/9465

DOI : $10.4000 /$ lidil.9465

ISSN : 1960-6052

\section{Éditeur}

UGA Éditions/Université Grenoble Alpes

\section{Édition imprimée}

ISBN : 978-2-37747-315-

ISSN : $1146-6480$

\section{Référence électronique}

Badreddine Hamma, «Présentation. - Le passif dans la langue parlée », Lidil [En ligne], 64 | 2021, mis en ligne le 01 novembre 2021, consulté le 25 novembre 2021. URL : http://journals.openedition.org/ lidil/9465; DOI : https://doi.org/10.4000/lidil.9465

Ce document a été généré automatiquement le 25 novembre 2021.

(C) Lidil 


\title{
Présentation. - Le passif dans la langue parlée
}

\author{
Badreddine Hamma
}

1 L'intérêt grandissant porté à l'oralité et au discours en interaction ces dernières années a ouvert de nouvelles perspectives dans la recherche en linguistique et a permis de faire des découvertes inédites, de l'ordre d'une vraie rupture épistémologique dans certains domaines des sciences du langage ou, du moins, d'une révolution au niveau de la nature des données utilisées et de la manière dont on peut les appréhender. Parmi les questions que l'oralité a permis de mettre en discussion, il y a la fameuse polémique sur la légitimité de la phrase pour décrire, analyser ou représenter le discours dans sa globalité, sans tomber dans l'exclusion de certains segments. C'est le cas des nominativus pendens (cf. Le Querler, 2003), qui, bien que faisant partie intégrante de ce qui est énoncé sur les plans morphosémantiques et pragmatiques, ne sont généralement pas régis par des nœuds phrastiques et se trouvent, de ce fait, mis sur la touche dans les méthodes traditionnelles. Ce débat autour de la pertinence de la phrase s'est progressivement érigé entre détracteurs (cf. entre autres, Blanche-Benveniste \& Jeanjean, 1987 ; Berrendonner \& Béguelin, 1989 ; Béguelin, 1999, 2002 ; Berrendonner, 2002 ; Groupe de Fribourg, 2012) et défenseurs (cf. entre autres, Kleiber, 2003 ; Wilmet, 2011; Le Goffic, 2016). Et bien que le débat n'ait pas abouti à la mise à l'écart de la phrase, vu qu'elle reste malgré tout opérationnelle sur le plan microsyntaxique, il a amené de nouvelles interrogations très constructives, faisant apparaitre que cette unité ne suffit pas pour rendre compte de l'énonçable dans sa labilité, dans ses nombreux enchevêtrements et dans sa grande variabilité. Aussi de nouveaux types de regroupements et de principes d'analyse ont-ils vu le jour dans le cadre d'une macrosyntaxe. Dans cette approche émergente, les groupes écartés traditionnellement trouvent toute leur place. L'étude du passif à partir de données orales dans différentes langues constitue une illustration très éloquente de cette transition et de ses enjeux. Et c'est ce dont il sera question dans le présent numéro consacré au passif à l'oral et à l'apport des corpus oraux à la description de ce tour. 


\section{Vers une exemplification diversifiée et plus réaliste}

2 Un tour d'horizon des différents travaux sur le passif, aussi bien dans les ouvrages de référence et de vulgarisation que dans la recherche spécialisée en linguistique ${ }^{1}$, permet de se rendre compte que ce tour n'a pas fait l'objet d'études significatives dans ses manifestations orales en français, hormis chez Blanche-Benveniste $(1988,2000)$, ou plus récemment chez Hamma (de 2015 à 2020b) et Druetta (2020). L'exemplification habituelle est faite essentiellement de structures simplifiées et de phrases formatées et quasi figées. On en trouve aujourd'hui des traces dans le discours didactique, aussi bien en FLE qu'en FLM, avec des exemples artificiels et usés, du type La souris est mangée par le chat $(\mathrm{B} 1,2015)^{2}$; La souris sera dévorée par le chat ; Le fromage a été mangé par la souris $\left(5^{\mathrm{e}}\right.$, $2010)^{3}$; Le chat a été nourri par Caston (Grevisse du collège, 2018) ${ }^{4}$; Les enfants sont élevés par les parents ; Le coupable est découvert par le détective $\left(5^{\mathrm{e}}, 2008\right)^{5}$. Ces illustrations utilisent, de fait, surtout des schémas actanciels dichotomiques stéréotypés, opposant deux forces, avec une relation décrite le plus souvent comme une action subie, du type chat / souris, maitre / chat, souris / fromage, détective / coupable, parents / enfants, etc. Notons, par ailleurs, que les arguments du procès passif, dans ce type d'exemples, sont généralement représentés par des syntagmes nominaux pleins (à noyau lexical), ce qui n'est guère représentatif, comme nous allons le voir plus loin, d'une langue comme le français. Ces exemples peuvent aussi reprendre des noms propres du patrimoine littéraire ou culturel après leur simplification et leur adaptation au point grammatical abordé (Candide fut élevé dans un beau château; Hänsel et Gretel ont été abandonnés dans la forêt... $)^{6}$. On retrouve aussi des exemples repris dans des écrits journalistiques, relatant tout type d'évènements en rapport avec l'actualité (Le corps d'un homme a été trouvé lundi sur le quai), ou aussi repris dans des écrits historiques, scientifiques ou techniques (La radioactivité a été découverte en 1896 ; L'Amérique a été découverte par Christophe Colomb ; Le calcium est absorbé par les os...). Les exemples cités par les linguistes et les grammairiens n'échappent pas à cette règle ${ }^{7}$ : dans les rares cas où ils ne sont pas forgés, ils relèvent généralement de séquences descriptives, à fonction informative suivant les schémas dichotomiques ci-dessus. Et le grand absent, dans l'étude du passif de manière générale, est le recours à une exemplification orale authentique, issue d'échanges non lus, non contrôlés et s'inscrivant dans une approche dialogique et interactionnelle.

3 Le recours exclusif aux formes canoniques de l'écrit (doublé par cette exclusion des formes orales dans l'enseignement de la grammaire) pourrait s'expliquer par différents facteurs : dans le discours scolaire, on a tendance à croire que l'utilisation de phrases simples et simplifiées est salutaire pour les élèves. Une telle tendance semble favorisée par les représentations communes, qui font de l'écrit un objet valorisant, fiable et, donc, que l'on préconise en toute circonstance. En face, il y a le discours oral, qui passe pour un objet instable, fluctuant, répétitif, peu esthétique et, donc, qu'il faudrait éviter, ou que l'on limite à certaines questions de vocabulaire en classe de langue, alors même que les linguistes travaillant sur les interactions discursives y trouvent des plus-values insoupçonnables. Un autre facteur explicatif serait lié au fait que le développement des outils techniques et technologiques permettant de traiter de la langue parlée n'a pu réaliser des percées significatives que récemment, depuis à peine deux décennies. De nos jours, on dispose de grands corpus, comme PFC, CLAPI, CFPP2000, ESLO, OFROM ${ }^{8}$, des outils de traitement du signal qui ne cessent de s'affiner, allant du recueil à 
l'analyse en passant par le traitement du signal, l'annotation, l'extraction et l'archivage ou la diffusion, ainsi que des plateformes pour les héberger et des consortiums pour fixer et débattre des bonnes pratiques à adopter lors du traitement des données dans un souci d'objectivité et d'interopérabilité. Mais comme cet intérêt pour l'oralité est relativement nouveau, tout comme pour les outils mobilisés, cela expliquerait pourquoi ces aspects sont généralement absents ou rudimentaires dans la formation des maitres et des formateurs de FLE.

4 Eu égard au caractère récent de l'intérêt pour l'oralité et pour le discours en interaction, il est tout à fait légitime, ainsi, de penser que, historiquement, la nature des données utilisées dans la tradition a eu des conséquences sur les méthodes employées et sur les gloses que l'on a pu associer au passif. Une telle réalité engage à effectuer un travail complémentaire d'approfondissement et de vérification sur les emplois de ce tour dans différents contextes de la langue parlée. C'est la raison d'être du présent numéro, qui a vocation à considérer ce que pourrait apporter l'oral à l'étude $\mathrm{du}$ passif et dont les formes de l'écrit ne permettent pas nécessairement de rendre compte. L'accent sera mis sur les domaines sensibles aux variations diamésiques dans le discours (voir § 2), à la fois en français et dans différentes langues. L'idée est de travailler sur des exemples dont la source d'énonciation ne coïncide plus avec un archétype, un locuteur fictif et idéalisé comme à l'accoutumée, mais qui sont pris en charge par un locuteur réel, qui est investi dans et par une situation de communication authentique, ne se réduisant pas à donner des informations sur le monde, souvent connues ou prédictibles.

\section{De quelques domaines sensibles aux variations diamésiques dans les emplois passifs}

5 Les « variations diamésiques » (cf. Hamma, 2019b, 2020a) renvoient à la manière dont les formulations peuvent changer en fonction des contraintes imposées par la nature du canal utilisé. . Cela désigne, en particulier, les arrangements et les réajustements qu'impose l'oral par opposition à l'écrit et inversement. Si le message oral est généralement produit sans préparation et s'inscrit dans des interactions directes et assez imprévisibles, le message écrit, quant à lui, est le plus souvent prémédité et asynchrone. Ces deux dernières propriétés de l'écrit font que l'encodeur dispose d'une certaine latitude temporelle et matérielle lui permettant de formuler d'une manière optimale son message, à l'abri des lapsus, des oublis, des hésitations, de l'émotion, des erreurs et des répétitions, ce qui peut laisser des traces dans un brouillon (se reporter à Gadet (1996) et à Jahandarie (1999) pour une distinction plus détaillée de chacun des deux types de message). Bien que s'inscrivant dans un continuum, ces deux pôles du canal peuvent diverger sensiblement dans la prise en charge d'un tour grammatical comme le passif. L'étude de ce tour, à partir de ses manifestations seulement à l'écrit ou à partir de données forgées, autorise ainsi de revoir certaines thèses faisant globalement l'objet d'un consensus aujourd'hui. Le réexamen pourrait concerner, entre autres, la hiérarchie informationnelle que contribue à mettre en place ce type de construction, à travers, en particulier, les hypothèses de "dégradation » ou d'« occultation » de l'agent, d'un côté, et de « promotion » et de " saillance » de l'objet, de l'autre. Les nouvelles données in situ invitent également à repenser ce que l'on dit 
sur ce tour du point de vue de sa forme, de son statut syntaxique globalement et de ses différentes fonctions discursives.

\subsection{Schéma actanciel et hiérarchie informationnelle dans les segments passifs}

6 Le passif renvoie globalement à une distribution particulière des rôles sémantiques dans leur relation avec un certain procès. C'est dans cet esprit que ce tour est le plus souvent ressenti et présenté dans la tradition comme le résultat d'une transformation, d'un retournement de l'ordre des actants. La tournure passive donnerait à voir alors une certaine "thématisation » impliquant, d'un côté, la "promotion» de l'objet du verbe au rang de sujet grammatical, ce qui est souvent associé dans la tradition à un certain effet de «saillance» ou de «focalisation» (§ 2.1.1.), de l'autre, l'« occultation » ou la «destitution » du rôle d'agent, ou, du moins, son extraposition (étant relégué à la fin de la phrase). C'est dans cet esprit que l'on parle, dans la vulgate, d'optionalité et de secondarité à propos du complément d'agent (\$ 2.1.2).

\subsubsection{Le passif et la notion de « saillance »}

7 La notion de «saillance» ou de "focalisation» que défendent de nombreux grammairiens et linguistes (Johnson-Laird, 1968; Givon, 1981; Descles \& Guentchéva, 1993 ; Gaatone, 1998 ; Riegel et coll., 1994 ; Creissels, 2001 ; Brahim, 2001, entre autres) serait un effet résultant de l'opération de thématisation de l'objet (argument postverbal) et de la périphérisation ou de la mise à l'écart concomitante de l'agent ("premier actant et sujet à l'actif»). L'élargissement aux données orales a, en revanche, permis de remettre en cause cette hypothèse (sur ce point, voir Hamma, 2015, 2017, 2020a, 2020b). Ainsi, interpréter cette "promotion" syntaxique du deuxième actant du verbe (devenant le No de la construction passive) comme un indice de supériorité informative de ce nouveau support de phrase (cf. Gaatone, 1998, p. 213, 230 et suivantes) aux dépens du premier actant du verbe parait contestable et trompeur. Ainsi, dans les corpus ESLO, il s'est avéré, par exemple, que le NO passif renvoie surtout à un pronom non accentué, dans $88,23 \%$ des cas relevés des verbes de parole (voir Hamma dans ce numéro) ; or, il est difficile de considérer qu'un proclitique est porteur d'une quelconque idée de mise en valeur ou de focalisation, étant un élément faible, à part quand il entre dans des dispositifs externes de focalisation (dislocation, accent contrastif, extraction, etc.), auquel cas, ce n'est plus l'affaire du passif. C'est l'un des revers d'une interprétation reposant exclusivement sur des phrases forgées, avec des actants lexicaux. De toute façon, dans cette optique transformationnelle, l'argument de la thématisation se trouve limité par les nombreuses impasses et "lacunes distributionnelles ", liées à la nature de la relation sémantique entre le procès et son argument postverbal (voir Jalenques (2015) et dans ce numéro).

\subsubsection{Le passif et la notion d'« occultation » de l'agent}

8 En dehors de quelques constructions marginales (semi-)figées (Mathieu, 1993), le complément d'agent est considéré quasi unanimement comme un segment facultatif et sacrifiable, soit à travers sa suppression possible, soit parce qu'un énoncé passif a 
surtout vocation à être "court " (à se construire sans complément d'agent). C'est, par exemple, le point de vue de Desclés et Guentchéva (1993, p.77 et 80), de Lamiroy (1993, p. 64) ou aussi de Brahim (1996), de Kahane (1998, p. 327) et de Mel'cuk (2001). Ce postulat constituerait le pendant et le résultat de la thématisation de l'objet (cf. supra) : on mettrait en avant l'objet aux dépens de l'agent. Encore une fois, le recours aux données orales parait démentir cette hypothèse au moins partiellement et ce, sur plusieurs plans. En effet, la précision d'un complément d'agent, dans un échange in situ, semble, d'après les données orales, l'indice d'une grande informativité. En témoignent les extraits suivants des ESLO où toute tentative de suppression des compléments d'agent parait impossible sans transgresser les lois du discours les plus élémentaires, comme celles de pertinence, de qualité ou de quantité (Grice, 1975). Le complément d'agent y semble particulièrement récalcitrant, car son absence pourrait générer une interprétation banale, qui serait de facto erronée et contradictoire avec ce qui aurait été affirmé :

(1)

J0304: quand on quand on voit certains certains textes euh qui ont été euh certaines paroles qui ont été dites par des ministres j'avoue vraiment que on peut un peu désespérer de l'avenir euh intellectuel des des enfants qui viendront

(2)

SD14 : et moi c'est tout à fait par hasard que que je suis revenu Oui

ch_OB1 : ah oui alors c'est c'est quoi ce hasard c'est?

SD14: c'est une petite annonce euh sur le Monde euh voilà comme ça j'ai été recruté euh sur une petite annonce du Monde par mon par mon directeur c'est je suis entré au CNRS comme ingénieur

(3)

FT07: et qu'est-ce que vous diriez à quelqu'un pour le convaincre d'aller voir ce film?

KF467: qu'est-ce qu'on pourrait dire à quelqu'un bah d'abord parce que c'est présenté par un ciné-club historique d'Orléans qui s'appelle l'Apac et qu'il faut aller voir les films que présente l'Apac et puis parce que bon il se trouve que c'est un très beau film sur euh

(4)

L1: une autre question euh c'est pas du tout politique mais enfin euh oui on a beaucoup parlé des évènements de mai juin euh les Anglais étaient évidemment pas là en France à ce moment-là est-ce que vous pourriez décrire enfin pour les Anglais qui vont écouter les bandes ce qui s'est passé en France à ce moment-là ?

L2 : difficile ce que vous me demandez là

L1 : hein? est-ce que vous pourriez décrire enfin ce qui s'est passé en France quoi? parce que vous savez que les bandes vont partir après en Angleterre qu'elles seront écoutées par les étudiants euh...

Dans tous ces exemples, il est difficile - pour ne pas dire impossible - de se passer du complément d'agent sans rendre les propos communiqués insensés et incohérents dans leurs contextes respectifs. Ainsi, ce que le locuteur met en avant, ce n'est sans doute pas le support-sujet passif (élément connu et déjà là, représenté dans ces exemples par de simples pronoms endophoriques ou exophoriques), mais bel et bien le complément d'agent en italique explicité (c'est l'information nouvelle, en addition au procès passif). En l'occurrence, on assiste à une opposition entre, d'un côté, un agent « singularisé » et très " saillant ", dans (1-4) : respectivement, par des ministres, par mon directeur, par un ciné-club historique..., par les étudiants, de l'autre, un agent " commis d'office " pour un procès donné et dont la précision est superflue (étant conforme à la doxa, aux savoirs partagés et à des situations typiques), et qui se trouve, ici, écarté par la précision de 
l'agent : des ministres s'opposant en l'occurrence à « une personne non instruite »; mon directeur de thèse à " un parfait inconnu »; un ciné-club historique à " un avis personnel banal » et les étudiants à « des usagers importuns » (pour une analyse plus détaillée, voir Hamma, 2015 et 2020b).

\subsubsection{Le statut syntaxique des segments passifs de l'oral}

Une simple comparaison des exemples classiques avec des énoncés pris dans des échanges authentiques permet de s'apercevoir que les passifs de l'oral ne suivent que très rarement le schéma syntaxique canonique (No être $V p p$ (par N1)), aussi bien au niveau du statut énonciatif global du segment passif (sur le plan macrosyntaxique), qu'au niveau de sa structure interne (sur le plan microsyntaxique). Observons les exemples suivants des ESLO (5-6) :

(5)

... mais mon fils leur a dit bah ça va fermer... et n'oubliez pas bande d'idiots il est il les a il est bien aimé par ses ouvriers il dit si vous cassez tout et puis... comment vous travaillerez demain.

(6) ... parce que je trouve dommage que des personnes qui sont entendues par des milliers de de Français euh parlent très mal.

11 Pour ne reprendre, dans cette brève présentation, que quelques-uns des aspects en jeu (voir Hamma dans ce numéro pour une étude plus détaillée) : le segment passif en italique dans ces exemples, d'une part, ne constitue pas le noyau énonciatif de l'échange, comme à l'accoutumée, où l'on a des énoncés autonomes et isolés, d'autre part, il n'y constitue même pas un énoncé à part entière. C'est aussi le cas des exemples (1) et (4) ci-dessus : on a une simple parenthétique, dans (5), une enchâssée relative, faisant office de complément de nom, dans (1) et (6), une enchâssée complétive ayant le statut d'un postnoyau, dans (4). Et c'est d'ailleurs pour cette raison que nous préférons parler ici de «segment passif » plutôt que de " phrase », ou d' « énoncé ». On remarquera aussi que le passif parait greffé ou surajouté dans ces exemples, comme le montre la possibilité de le supprimer intégralement dans certains cas, sans affecter la grammaticalité de l'énoncé-hôte, dans (1) et (4-6). Ceci dit, le passif dans tous ces exemples est dicté par des besoins d'interaction (le locuteur réagit à ce qu'il voit ou entend) et s'avère indispensable du point de vue pragmatique.

\subsubsection{Diversité des formes passives à l'oral}

Dans une définition restrictive de ce qu'est un passif, cette appellation est couramment réservée aux formes, dites "périphrastiques ", « synthétiques » ou " analytiques » (cf. Gaatone, 1998) et que l'on considère comme la forme "canonique " (combinant l'auxiliaire être et le participe passé d'un certain type de verbes). C'est d'ailleurs cette forme que l'on privilégie dans le discours scolaire, aux dépens des autres formes possibles, pouvant dévier de ce schéma, et que l'on appelle parfois «non canoniques » (cf. passifs pronominaux, factitifs, adjectivaux, nominaux, converses, etc.). Ce dernier type de constructions semble pourtant remplir les mêmes fonctions discursives rappelées ci-dessus en rapport avec la hiérarchie informationnelle et ont une fréquence dépassant parfois les formes canoniques dans l'usage: d'après une sélection de 1809036 mots des ESLO $^{10}$ (cf. Hamma et coll., 2017), ces passifs non canoniques 
représentent globalement $52 \%$ des emplois observables sur un total de 3989 énoncés passifs. De fait, leur prise en compte s'avère particulièrement utile dans ce numéro.

13 De toute façon, dès lors que l'on considère la question du passif dans une perspective contrastive et translinguistique, il devient inévitable d'étendre les constructions étudiées aux autres manifestations de la diathèse passive, tant qu'une distribution concurrente active ou non marquée est attestée ou aurait été possible. Cet élargissement s'avère indispensable aussi quand on sait que, d'une part, dans certaines langues, l'un des composants-clés (l'auxiliaire être) n'a pas d'existence matérielle et peut prendre d'autres formes (cf. l'arabe cairote dans ce numéro) ou alors peut se dédoubler, comme en espagnol, avec les formes ser et estar ou aussi connaitre plusieurs concurrents, comme en italien, où l'expression du passif est très fréquente avec d'autres (semi-)auxiliaires, comme venire, andare, rimanere, restare (cf. Martinot \& Gerolimich et Castellani dans ce numéro). D'autre part, si l'on considère certains phénomènes de traduction, on pourrait voir que la notion de passif peut se diluer dans d'autres constructions ou paraphrases complètement divergentes. Ainsi, la traduction hongroise du passif périphrastique du français, avec un agent, peut se faire à travers certaines formes actives, comme le montre l'étude de Sörés dans ce numéro. Par ailleurs, l'idée de "passif» est parfois récupérée par d'autres procédés de thématisation, comme c'est le cas en roumain parlé (cf. Pop dans ce numéro).

\section{Les contributions de ce numéro}

Ce numéro réunit une série de huit textes sur le fonctionnement du passif dans diverses langues parlées (français orléanais, italien, hongrois, roumain, arabe cairote et capverdien santiagais) et prend une dimension contrastive dans quatre de ces études en relation avec le français (français-italien; italien-français; français-hongrois et roumain-français). Notons que les données orales utilisées dans ces différentes contributions se situent à différents niveaux d'un gradient de spontanéité et de formalité, selon les contextes et les conditions de recueil (échanges libres, repas, sorties, enquêtes de terrain, sollicitation et restitution dans des tâches scolaires, etc.).

Ce numéro se propose donc de faire le point sur les apports de l'oralité et des corpus oraux à l'étude et à la description du passif dans différentes langues, aussi bien sur le plan de la forme que sur le plan des interprétations et des usages possibles. Les résultats et analyses exposés ici ont ainsi vocation à être originaux et inédits, du moins en ce qui concerne le passif et les différents aspects et problèmes y afférents.

16 Ainsi, Badreddine Hamma interrogera le statut grammatical global du passif périphrastique à partir d'un corpus de verbes de parole extrait des ESLO 1 \& 2. Cette étude vient discuter un présupposé théorique largement partagé, selon lequel le passif est coextensif à l'espace phrastique. Il y sera démontré qu'un segment passif ne représente pas toujours l'objet central de l'échange et qu'il peut renvoyer à un simple élément oblique dépendant d'un noyau énonciatif (segment macrosyntaxique) ou d'une tête syntagmatique donnée (segment microsyntaxique). La démarche adoptée s'inspire de certains principes théoriques des écoles aixoise et fribourgeoise.

17 Dans une démarche contrastive, Claire Martinot et Sonia Gerolimich se pencheront sur la question de l'acquisition du passif dans différentes configurations morphosyntaxiques. L'étude repose sur des productions orales d'enfants francophones 
et italophones de 4 à 10 ans dans le cadre d'une tâche de restitution d'histoire. Il en ressort, par exemple, que les passifs périphrastiques sont beaucoup moins fréquents dans les deux langues que les passifs non canoniques, en particulier, les pronominaux et les formes en ilya/ $c^{\prime}$-emplois généralement marginalisés dans la littérature. De même, il y apparait que les enfants avant l'âge de 8 ans ne parviennent pas à réconcilier le rôle agentif du complément d'agent avec un sémantisme passif.

En partant de productions orales renfermant des passifs en français et après les avoir soumises à une traduction en hongrois, Anna Sörés s'intéressera, dans une perspective contrastive, au phénomène de "détransitivisation». Les exemples sont repris essentiellement des ESLO 1 \& 2. L'auteur défend l'hypothèse selon laquelle les passifs sans complément d'agent ou de cause se rapprochent davantage de la voix moyenne (des se-verbes). L'étude aboutit à une classification des emplois passifs à l'oral dans les deux langues et où la présence ou non d'un complément d'agent constitue un critère crucial. En l'absence d'agent, il est possible, selon l'auteur, de parler d'un certain effet de « saillance ", qui affecte le procès globalement et non l'objet, contrairement à ce qui est souvent répété dans la littérature sur le passif.

Julie Haslé se propose d'étudier les formes verbales suivant le moule itCaCaC d'une langue vernaculaire sémitique: l'arabe du Caire. Dans cette langue, l'idée de passif est marquée morphologiquement à travers le préfixe ( $\mathrm{t}-\mathrm{I} »)$ et par un schéma dérivationnel non concaténatif (ici, le schème consonantique). Le corpus employé provient d'enregistrements effectués par l'auteur et relève de différents genres d'oral (échanges libres lors de repas, des réunions entre amis, des sorties ou aussi des contes oraux pour enfants). En s'appuyant sur des critères sémantiques précis, l'auteur distingue trois interprétations possibles des formes retenues : passive, anticausative ou les deux, qu'elle met en relation avec la nature des rôles sémantiques "patient» ou " expérienceur".

20 À partir de l'observation d'une série de huit verbes sélectionnés dans les enquêtes orléanaises, ESLO 1 \& 2 (cf. recevoir, subir, supporter, arrêter, habiter, comporter, composer, constituer), Pierre Jalenques s'attache à expliquer certaines lacunes distributionnelles de ces verbes à l'oral dans leur usage au passif périphrastique. Il en ressort que certains emplois de ces verbes ne sont pas passivables (cf. arrêter un suspect vs arrêter l'école). L'auteur explique les impossibilités observées par ce qu'il appelle «variation de portée sémantique ». L'intérêt de l'étude réside aussi dans le fait de donner des arguments supplémentaires contre les thèses considérant que le passif et l'actif sont synonymes.

21 Liana Pop se consacre à l'étude du passif dans une " perspective graduelle » et ce dans différentes configurations morphosyntaxiques en roumain parlé. Elle y traite à la fois des formes canoniques et des formes non canoniques. Le corpus oral utilisé est l'IVLRA (Interaç̧iunea verbală în limba română actuală. Corpus (selectiv)). L'étude aboutit à la nécessité d'étendre ce que l'on appelle passif en roumain à d'autres formes moins conventionnelles, d'ailleurs non enregistrées dans les grammaires traditionnelles, dont certaines tournures lexicales ou aussi certaines opérations de thématisation, qui peuvent être assimilées, selon l'auteur, à des constructions passives.

Dans le cadre d'une comparaison des usages du passif à l'écrit et à l'oral en italien, Cristina Castellani tentera d'explorer les propriétés de chaque genre de discours et de cerner l'apport de l'oralité à la description de ce tour. Le corpus d'observation est fait pour l'essentiel de productions, d'abord orales, puis écrites, suscitées par certaines techniques de sollicitation particulières, visant à restituer et à commenter une 
sélection de vidéos visionnées par les étudiants. L'auteur s'y intéresse aux changements observables de l'emploi des auxiliaires essere, venire, andare et de leurs propriétés distributionnelles. L'un des résultats obtenus - et qui va à l'encontre de ce qui est couramment admis - concerne la supériorité en nombre des occurrences passives à l'oral en italien en comparaison avec l'écrit dans le corpus considéré.

Le huitième texte de ce numéro offre une étude qualitative et quantitative sur une langue vernaculaire très peu décrite et encore moins à partir de données orales: le capverdien santiagais, un créole à base portugaise. Cette recherche est conduite par Quint \& Vieira de la collecte des données sur le terrain, à leur analyse morphosyntaxique en passant par leur annotation. Le corpus est formé de douze contes traditionnels du Cap-Vert appartenant au genre oral. Les auteurs s'y intéressent aux propriétés distributionnelles du procès au passif, à la fois en rapport avec les marques de temps, d'aspect et de polarité, ou aussi en rapport avec les participants qu'il peut sélectionner.

\section{BIBLIOGRAPHIE}

ATTAL, Pierre. (1985). Le passif : étude de corpus. L'information grammaticale, 27, 10-13.

BÉGUELIN, Marie-José. (1999). De la phrase aux énoncés : bilan et perspectives. Travaux neuchâtelois de linguistique, 31, 143-153.

BÉGUELIN, Marie-José. (2002). Clause, période ou autre ? La phrase graphique et la question des niveaux d'analyse. Verbum, XXIV(1-2), 85-108.

BERRENDONNER, Alain. (2002). Les deux syntaxes. Verbum, XXIV(1-2), 23-36.

BERRENDONNER, Alain \& REICHLER-BÉGUELIN, Marie-José (1989). Décalages : les niveaux de l'analyse linguistique. Langue française, 81 , Structurations de textes : connecteurs et démarcations graphiques, 99-125.

BLANCHE-BENVENISTE, Claire. (1988). La notion de contexte dans l'analyse syntaxique des productions orales : exemples des verbes actifs et passifs. Recherches sur le français parlé, 8, 39-57.

BlAnCHE-BENVENISTE, Claire. (2000). Analyse de deux types de passifs dans les productions de français parlé. Dans L. Schoesler (dir.), Le passif : actes de colloque (Études romanes, 45, p. 303-319). Museum Tusculanum Press.

BlanCHE-BENVENISTE, Claire \& JEANJEAN, Colette. (1987). Le français parlé : transcription et édition. INALF.

BRAHIM, Ahmed. (1996). L'occultif: hypothèse pour un traitement trans-linguistique du " passif » et des structures apparentées. Association tunisienne de linguistique.

BraHIM, Ahmed. (2001). Passif et moyen dans les langues du pourtour méditerranéen. Linx, 45, 107-116.

CREISSELS, Denis. (2001). Remarques sur la notion de passif et l'origine des constructions passives. Linx, 45, 71-82. 
DESClÉs, Jean-Pierre \& GUENTCHÉVA, Zlatka. (1993). Le passif dans le système des voix du français. Langages, 27, 73-102.

DRUETTA, Ruggero. (2020). Le passif à l'oral. Phénoménologie et propriétés aspectuelles dans OFROM. Studia linguistica romanica, 4, 150-174.

DuBoIs, Jean. (1967). Grammaire structurale du français. Le verbe. Larousse.

GAATONE, David. (1998). Le passif en français. Duculot.

GADET, Françoise. (1996). Une distinction bien fragile : oral/écrit. Travaux neuchâtelois de linguistique, 25, 13-27.

GivóN, Talmy. (1981). Typology and Functional Domains. Studies Language, 5(2), 163-193.

GRICE, Herbert Paul. (1975). Logic and Conversation (trad. 1979). Éditions du Seuil.

GRoss, Maurice. (1993). Un nouvel agent en par. Langages, 109, Sur le passif, 32-34.

GROUPE DE FRIBOURG. (2012). Grammaire de la période. Peter Lang.

HAMMA, Badreddine. (2015). Agent passif en par et sujet actif : les dessous d'un contraste. Revue de sémantique et pragmatique, 37, 61-83.

HAMma, Badreddine. (2017). Tentative de classification des « compléments d'agent » dans les phrases passives achevées et dans les énoncés longs à sens passif. ELA, 187(3), 311-324.

HAMMA, Badreddine. (2019a). Quand l'interaction n'est pas là, la souris est mangée par le chat ! Remarques sur l'enseignement du passif en classe de français. Dans A.-S. Calinon, B. Hamma, K. Ploog \& M. Skrovec (dir.), Linguistique interactionnelle, grammaire de l'oral et didactique du français (p. 237-262). Presses universitaires de Franche-Comté.

HAMMA, Badreddine. (2019b), Pour une didactique de la diamésie : revers du recours à la phrase forgée dans l'enseignement (cas de la litote et du passif). Dans C. Avezard-Roger, C. Corteel, J. Goes \& B. Lavieu-Gwozdz (dir.), La phrase : carrefour linguistique et didactique (p. 281-301). Artois Presses Université.

HAMMA, Badreddine. (2020a), Variations diamésiques autour du passif périphrastique en français. Dans M. Saiz-Sánchez, A. Rodríguez Somolinos \& S. Gómez-Jordana Ferary (dir.), Marques d'oralité et représentation de l'oral en français (p. 43-66). Presses universitaires Savoie Mont Blanc.

HAMMA, Badreddine. (2020b). Pourquoi ne peut-on pas se passer de «l'agent passif » dans une vraie conversation? Dans J. Chaker, L. Oueslati \& L. Hosni (dir.), Le dialogue et la conversation à la croisée des approches (p. 87-103). Latrach Édition.

HAMMA, Badreddine. (2020c). Les figures de l'agent et du complément d'agent dans l'usage et dans le métalangage. Dans M. Meulleman, S. Palma \& A. Theissen (dir.), Liber Amicorum. Mélanges offerts à Emilia Hilgert (p. 147-272). Éditions et Presses universitaires de Reims (Épure).

HAMMA, Badreddine. (2020d). Le passif enseigné sur fond de crise politique : exemple des évènements de Mai 68 à Orléans. Le Langage et l'Homme, 551, Apprentissage des langues : compétence pragmatique, interculturalité, 149-164.

HAMMA, Badreddine. (2020e). Aborder la diathèse passive en contextes de français langue étrangère par la voie de l'oralité. Dans P. Larrivée \& F. Lefeuvre (dir.), La didactisation du français vernaculaire (p. 68-111). Presses universitaires de Caen.

HAMMA, Baderddine, TARDIF, Amélie \& BADIN, Flora. (2017). Le passif à l'oral. Fiche du Projet FRACOV (Français contemporain vernaculaire) : <www.ortolang.fr/market/corpora/fracov?path=\%2F>. 
HELLAND, Hans Petter. (2002). Le passif périphrastique en français moderne. Museum Tusculanum Press.

JAHANDARIE, Khosrow. (1999). Spoken and Written Discourse: A Multidisciplinary Perspective. Greenwood Publishing Group.

JALENQUES, Pierre. (2015). Le passif en français et les contraintes distributionnelles des verbes. Corela, 13(1). <https://doi.org/10.4000/corela.4015>.

JoHnSON-LAIRD, Philip. (1968). The Interpretation of the Passive Voice. Quarterly Journal of Experimental Psychology, 20, 69-73.

KAHANE, Sylvain. (1998). Le calcul des voix grammaticales. Bulletin de la Société de linguistique, 93, 325-348.

KLEIBER, Georges. (2003). Faut-il dire adieu à la phrase ? L'information grammaticale, 98, 17-22.

LAGAE, Véronique. (2005). Les formes en être + participe passé à valeur résultative dans le système verbal français. Cahiers Chronos, 12, 125-142.

LAmiroy, Béatrice. (1993). Pourquoi il y a deux passifs. Langages, 109, Sur le passif, 53-72.

LE GoffIC, Pierre. (2016). «Y a-t-il une syntaxe au-delà de la phrase ? » Retour sur une question lancinante. Dans L. Sarda, D. Vigier \& B. Combettes (dir.), Connexion et indexation : ces liens qui tissent le texte. ENS Éditions.

LE QUERLER, Nicole. (2003). Le nominativus pendens en français. Cahiers de praxématique, 40, 149-166. MATHIEU, Yvette Yannick. (1993). Quelques passifs avec agent obligatoire. Langages, 109, Sur le passif, 35-36.

Mel'cuK, Igor. (2001). Grammatical Voice French (A Short Description in the Meaning-Text Framework). Dans V. S. Chrakovskij, M. Grochowski \& G. Hentschel (dir.), Studies on the Syntax and Semantics of Slavonic Languages (p. 265-290). Universität Oldenburg.

MionI, Alberto. (1983). Italiano tendenziale: osservazioni su alcnia spetti della standardizzazione. Dans P. Benincà \& G. B. Pellegrini (dir.), Scritti linguistici in onore di Giovan Battista Pellegrini (vol. 1, p. 495-517). Pacini.

PAUZE, Isabel. (2001). Les fonctions textuelles de la voix passive (Thèse de doctorat). Université Lyon 2. RAPATEL, Philippe. (2010). À la recherche de l'agent masque : l'argentinité implicite. Cahiers du Laboratoire de recherche sur le langage, 4, Construction de la cohérence, construction du sens, 1-9. RieGEL, Martin, Pellat, Jean-Christophe \& Rioul, René. (1994-2009). Grammaire méthodique du français. Presses universitaires de France.

SöRÉs, Anna. (2006). Le passif : constructions non prototypiques. Dans A. Sörés, Le hongrois dans la typologie des langues (p. 105-130). Lambert-Lucas.

WILMET, Marc. (2011). Plaidoyer pour la phrase graphique. Dans G. Corminboeuf \& M.-J. Béguelin (dir.), Du système linguistique aux actions langagières (p. 221-234). De Boeck-Duculot.

\section{NOTES}

1. Voir, entre autres, Attal (1985), Dubois (1967), Comrie (1988), Gross (1993), Brahim (1996, 2001), Gaatone (1998), Mel'cuk (2001), Pauze (2001), Helland (2002), Lagae (2005), Sörés (2006) et Rapatel (2010). 
2. Cocton, M.-N. (2015). Saison 3. Méthode de français, B1. Didier.

3. Beltrando, B. (2010). L'atelier de langage, $5^{e}$. Éditions Hatier.

4. Gaillard, B. et coll. (2018). Grevisse du collège. Magnard.

5. Potelet, H. (2008). Français livre unique, $5^{e}$. Éditions Hatier.

6. La connaissance préalable de l'œuvre ou des personnages représentés par les actants peut parfois permettre de faire l'économie de certaines précisions sur le contexte (Julien Sorel, Frédéric Moreau, Mme Bovary, Don Juan, Meursault ou aussi Tarzan, Zorro...).

7. Pour plus de détails sur ce point, se reporter à Hamma (2019a, 2019b et 2020a).

8. PFC (Phonologie du français contemporain), CLAPI (Corpus de langue parlée en interaction), CFPP2000 (Corpus de français parlé parisien des années 2000), ESLO (Enquêtes sociolinguistiques à Orléans), OFROM (corpus Oral du français de Suisse romande).

9. On doit le terme « diamésie » à Mioni (1983, p. 508). L'auteur l'utilise pour distinguer l'italien standard par opposition aux variations régionales. De nos jours, cette appellation renvoie surtout à la variation de canal en ciblant l'opposition écrit-oral et est parfois utilisée chez certains auteurs comme synonyme de la variation diaphasique.

10. ESLO renvoie aux Enquêtes sociolinguistiques à Orléans, un grand corpus oral réalisé dans le cadre des activités du Laboratoire ligérien de linguistique. Il est en libre accès à l'adresse $<$ www.eslo.huma-num.fr> et sur la plateforme Cocoon: <https://cocoon.huma-num.fr/exist/ crdo/collection_eslo.htm>.

\section{AUTEUR}

\section{BADREDDINE HAMMA}

LLL, UMR 7270, Université d'Orléans badreddine.hamma@univ-orleans.fr 